\title{
L-MATRICES WITH LACUNARY COEFFICIENTS
}

\author{
Ludovick Bouthat AND JaVAd Mashreghi*
}

Abstract. We show that an $L$-matrices $A=\left[a_{n}\right]$, with lacunary coefficients $\left(a_{n}\right)$ is a bounded operator on $\ell^{2}$, provided that $\left(a_{n}\right)$ satisfy an explicit decay rate. Moreover, by a concrete example, we see that the decay restriction is optimal. The extension to operators on $\ell^{p}$ spaces, for $p>1$, is also discussed.

Mathematics subject classification (2020): 15A60, 15A04, 39B42.

Keywords and phrases: Operator norm, sequence spaces, infinite matrices.

\section{REFERENCES}

[1] Ludovick Bouthat And Javad Mashreghi, The norm of an infinite L-matrix, Operators and Matrices, to appear, pages $1-12$.

[2] Durmuş Bozkurt, On the $l_{p}$ norms of Hadamard product of Cauchy-Toeplitz and Cauchy-Hankel matrices, Linear and Multilinear Algebra, 45 (4): 333-339, 1999.

[3] Arlen Brown, P. R. Halmos And A. L. Shields,, Cesàro operators, Acta Sci. Math. (Szeged), 26: 125-137, 1965.

[4] Man Duen Choi, Tricks or treats with the Hilbert matrix, Amer. Math. Monthly, 90 (5): 301-312, 1983.

[5] Dan Dai, Mourad E. H. Ismail and Xiang-Sheng Wang, Doubly infinite Jacobi matrices revisited: resolvent and spectral measure, Adv. Math., 343: 157-192, 2019.

[6] Pratibha G. Ghatage, On the spectrum of the Bergman-Hilbert matrix, Linear Algebra Appl., 97: 57-63, 1987.

[7] Paul Richard Halmos, A Hilbert space problem book, volume 19 of Graduate Texts in Mathematics, Springer-Verlag, New York-Berlin, second edition, 1982. Encyclopedia of Mathematics and its Applications, 17.

[8] G. H. Hardy, Divergent series, Éditions Jacques Gabay, Sceaux, 1992. With a preface by J. E. Littlewood and a note by L. S. Bosanquet, Reprint of the revised (1963) edition.

[9] David Hilbert, Ein Beitrag zur Theorie des Legendre'schen Polynoms, Acta Math., 18 (1): 155$159,1894$.

[10] Mourad E. H. ISMAIL AND FrantIŠEK ŠTAMPACH, Spectral analysis of two doubly infinite Jacobi matrices with exponential entries, J. Funct. Anal., 276 (6): 1681-1716, 2019.

[11] JAVAD MASHREGHI, Representation theorems in Hardy spaces, volume 74 of London Mathematical Society Student Texts, Cambridge University Press, Cambridge, 2009.

[12] JAVAD MASHREGHI AND THOMAS RANSFORD, Linear polynomial approximation schemes in Banach holomorphic function spaces, 9 (2): 899-905, 2019.

[13] JOHN LindSAY ORR, An estimate on the norm of the product of infinite block operator matrices, J. Combin. Theory Ser. A, 63 (2): 195-209, 1993.

[14] SÜleyman SolaK, Research problem: on the norms of infinite Cauchy-Toeplitz-plus-CauchyHankel matrices, Linear Multilinear Algebra, 54 (6): 397-398, 2006.

[15] SÜleyman Solak and Durmuş Bozkurt, On the spectral norms of Cauchy-Toeplitz and Cauchy-Hankel matrices, Appl. Math. Comput., 140 (2-3): 231-238, 2003.

[16] C. V. M. VAN DER MEE AND S. SEATZU, A method for generating infinite positive self-adjoint test matrices and Riesz bases, SIAM J. Matrix Anal. Appl., 26 (4): 1132-1149, 2005. 
[17] FrantišEK ŠTAMPACh ANd PAVEL ŠŤOvíčEK,, Spectral representation of some weighted Hankel matrices and orthogonal polynomials from the Askey scheme, J. Math. Anal. Appl., 472 (1): 483-509, 2019.

[18] H. RoopaeI, Factorization of the Hilbert matrix based on Cesàro and gamma matrices, Results Math., 75 (1), Paper No. 3, 12, 2020. 